\title{
Hereditary Multiple Exostoses Complicated By Common Peroneal Nerve Palsy
}

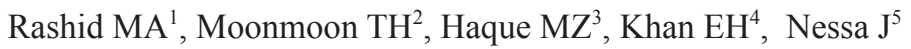

\begin{abstract}
Hereditary multiple exostoses is an autosomal dominant skeletal disorder. It is characterized by multiple bony prominences and skeletal deformities. It can lead to a series of complications including deformity of the joint, fractures through the tumor pedicle, mechanical block of nearby joints, nerve compression and malignant change. In this case it has been described a rare case of a seventeen-year old male patient with a history of hereditary multiple exostoses presented with foot drop. The management and Physiatric approach are discussed.
\end{abstract}

Key words: Hereditary multiple exostoses, foot drop, common peroneal nerve palsy

\section{Introduction}

Hereditary multiple exostoses (HME) is an autosomal dominant disorder characterized by multiple bony tumors or hamartomas arising near joints. These are cartilage capped and behave in a benign way and are associated with skeletal deformities ${ }^{1}$. It is the most common skeletal dysplasia, with a frequency of about $1: 18,000^{2}$. It develops in early childhood and becomes obvious with skeletal development ${ }^{2}$. The knees, hips, ankles, and shoulders are the most commonly affected sites and growth disturbances are often present, primarily in the forearms and legs ${ }^{3}$. Foot drop is not an uncommon complication which can occur due to compression neuropathy by the exostosis ${ }^{4}$.

\section{Case Report}

A seventeen year old male patient attended the outpatient department of Physical Medicine and Rehabilitation at Shaheed Suhrawardy Medical College Hospital (ShSMCH) with the complaints of difficulty in walking and lifting right foot for 1 month. The patiant also complained of multiple swelling around knees, shoulder, ankle joints, also in ribs and right scapular region. Some of the swellings were painful. Those were small initially and the patient could do the daily activities. However the swellings had increased in size over the last few years and the patiant was unable to fully extend the right knee and dorsiflex the right ankle. the patient underwent a surgical excision of a hard lump in the medial aspect of lower right thigh few months back. On physical examination there was a surgical scar mark at the supero-medial aspect of right knee (Fig-1).

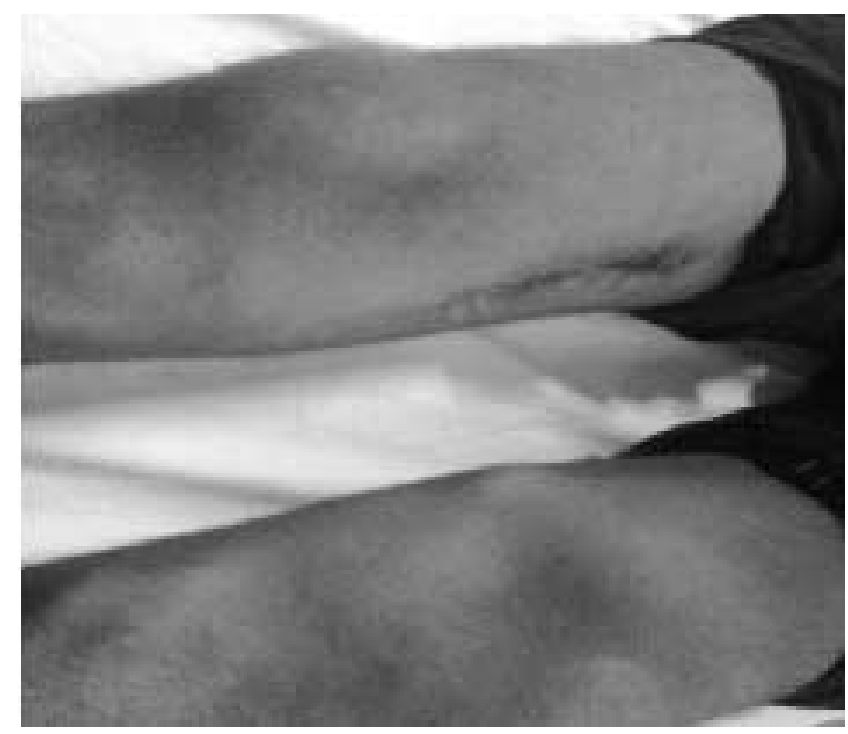

Fig-1: Multiple swelling around both knees with a surgical scar at supero-medial aspect of right knee.

There were multiple hard lumps around the knee joints with mild valgus deformity of the right knee as well as right ankle joints. There was also difficulty in knee extension and dorsiflexion of right foot but the peripheral pulses were felt normally. Sensation was impaired at the dorsum of right foot. Neurological examination revealed weakness of the tibialis anterior, lateral peroneal, and

1. Prof. MA Rashid, Professor \& Head, Department of Physical Medicine \& Rehabilitation, Shaheed Suhrawardy Medical College, Dhaka

2. Dr. Taslima Hoq Moonmoon, Assistant Professor, Department of Physical Medicine \& Rehabilitation, Shaheed Suhrawardy Medical College, Dhaka

3. Dr. Ziaul Haque, Assistant Professor, Department of Radiology, Bangabandhu Sheikh Mujib Medical University, Dhaka

4. Dr. Ehsanul Haque Khan, Assistant Professor, Department of Physical Medicine \& Rehabilitation, Shaheed Suhrawardy Medical College, Dhaka

5. Dr. Jebun Nessa, Assistant Professor, Department of Physical Medicine \& Rehabilitation, Shaheed Suhrawardy Medical College, Dhaka

\section{Correspondence}

Dr. Taslima Hoq Moonmoon, Assistant Professor, Department of Physical Medicine \& Rehabilitation, Shaheed Suhrawardy Medical College, Dhaka, Bangladesh; Email: taslimahoq@yahoo.com; Mobile: +8801715013612 
extensor digitorum muscles. Routine biochemical investigations were done which were found normal. Plain X-ray films showed multiple bony exostoses arising from the proximal end of both tibia and fibula at the both upper and lower end of femur, and alos humerus and scapula. The margins were smooth, internal trabeculations were observed. The cortices and marrow of the parent bone were in continuity with the growth (Fig-2,3).

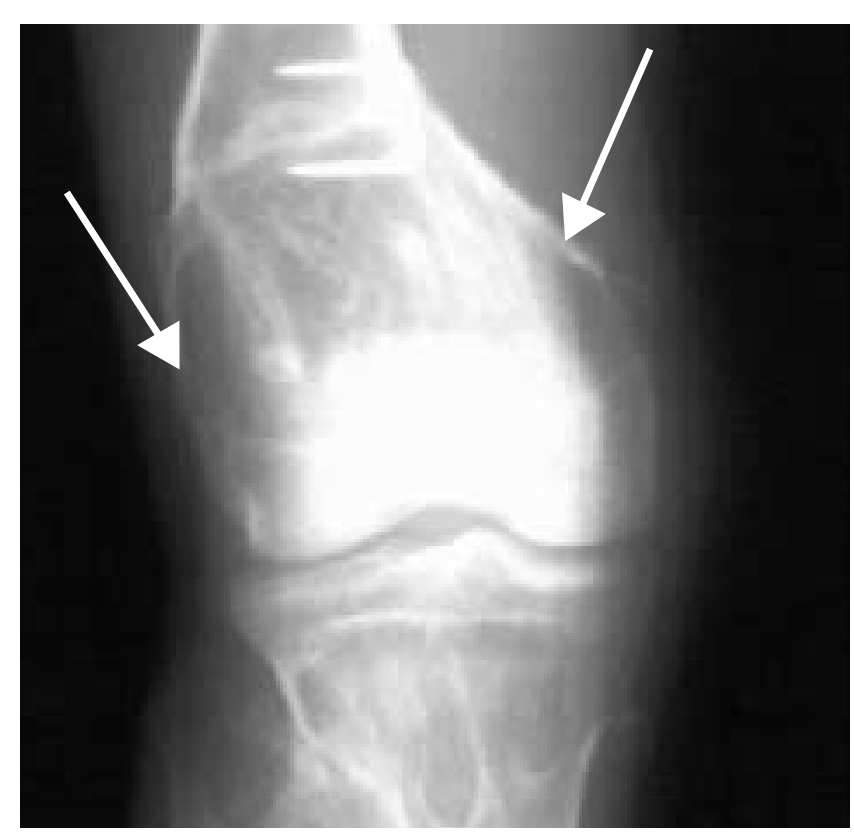

Fig-2: X-ray of right knee AP view multiple exostosis in upper end of tibia,fibula and lower end of femur with a surgical clip.

Nerve conduction study was done which revealed conduction block at the site of neck of fibula \& distal to that site. Electrophysiological studies confirmed denervation of the muscles supplied by the right common peroneal nerve. $\mathrm{CT}$ scan was done with $3 \mathrm{D}$ reconstruction which showed multiple bony outgrowth in metaphyseal region pointing away from the joint which was consistent with bony exostosis. CT scan delineated the extent of the lesion and also established unequivocally the continuity of cancellous portions of the lesion and the host bone. These characteristics distinguished this lesion from the occasionally similar-appearing bone masses of osteoma, juxtacortical osteosarcoma, soft tissue osteosarcoma, and juxtacortical myositis ossificans (Fig.4). MRI of right knee was done which showed multiple exostosis in proximal end of tibia and fibula and also in distal end of right femur. The cartilaginous cap showed a high signal intensity on T2weighted sequences. A narrow band of low signal intensity surrounding the cap representing the perichondrium was also seen (Fig-5). Based on history, physical examination, and different imaging modalities a diagnosis of foot drop in a case of HME was confidently made. The patient was managed accordingly by the drugs, physical therapy and orthosis. To relieve the pain, oral analgesic was used. In physical therapy electrical stimulation was given in right leg.

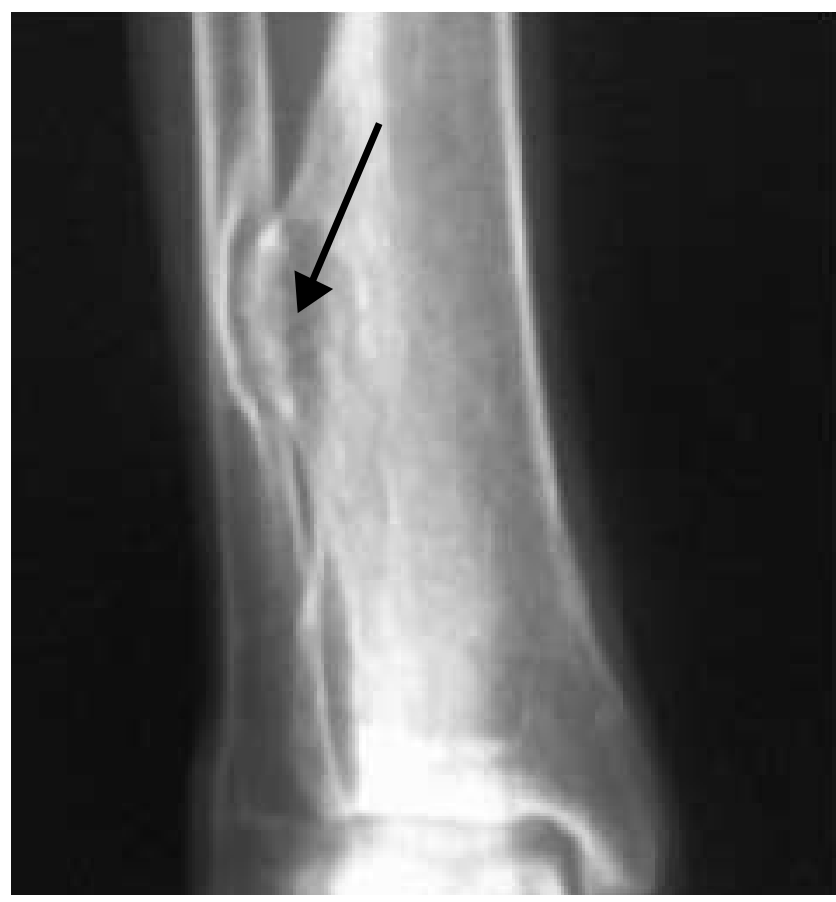

Fig-3: X-ray of right ankle AP view shows bony exostosis in distal end of tibia causing pressure erosion at the adjacent fibula.

Different exercises such as strengthening exercise, and range of motion exercise were prescribed. Wax bath was also applied to the right knee followed by stretching exercise to reduce the flexion deformity.

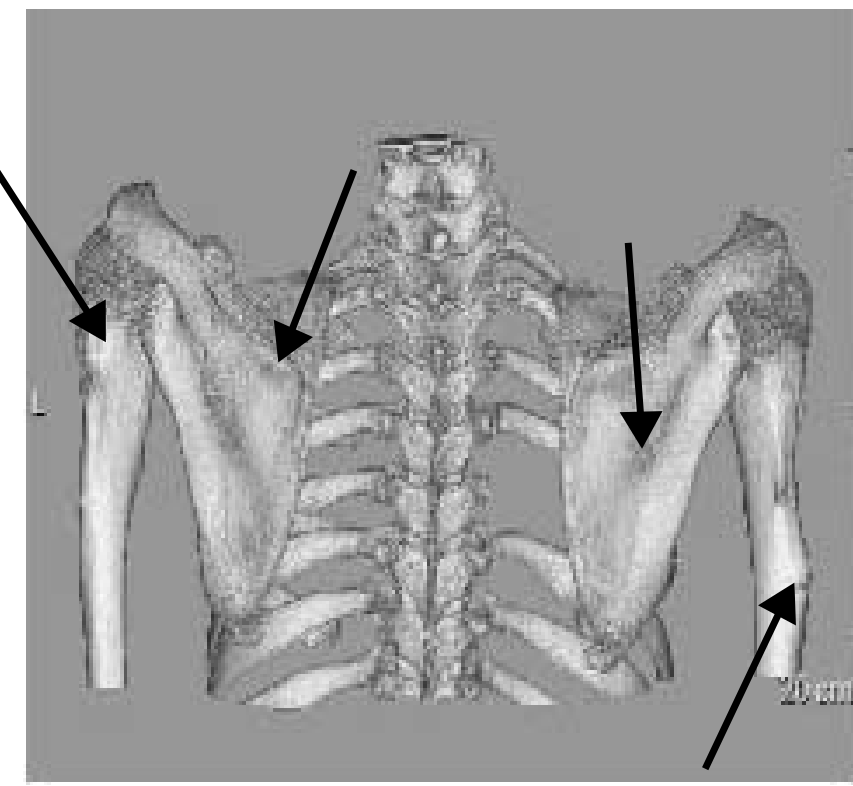

Fig-4: 3D reconstruction of CT scan of both shoulders showing multiple exostosis in proximal part of both humerus.

The patient was advised crutch, walking stick, and ankle foot orthosis subsequently as per requirement along with Gait training. The patient came to the department on wheel 
chair. During follow-up this condition improved gradually. Patient regained full range of right knee movement and significant recovery of the foot drop.

\section{Discussion}

Hereditary multiple exostoses constitute an autosomal dominant condition with variable penetrance ${ }^{5}$. In this disease, osteochondroma of many bones are caused by an anomaly of skeletal development ${ }^{6}$. The disease occurs only about $5 \%$ to $10 \%$ as often as solitary osteochondroma and is more common in males ${ }^{7}$. Approximately $66 \%$ of affected individuals have a positive family history ${ }^{7}$. The specific genetic abnormalities have recently been detected, with three distinct loci on chromosomes 8, 11, and 195. The lesions are usually discovered at about 2 years of age. The knees, hips, ankles, and shoulders are the most commonly affected sites and growth disturbances are often present, primarily in the forearms and $\operatorname{legs}^{3}$. Malignant degeneration is extremely rare ${ }^{7}$. Osteochondromas can lead to a series of complications including fractures through the tumor pedicle, mechanical block of nearby joints, and nerve compression ${ }^{8}$.

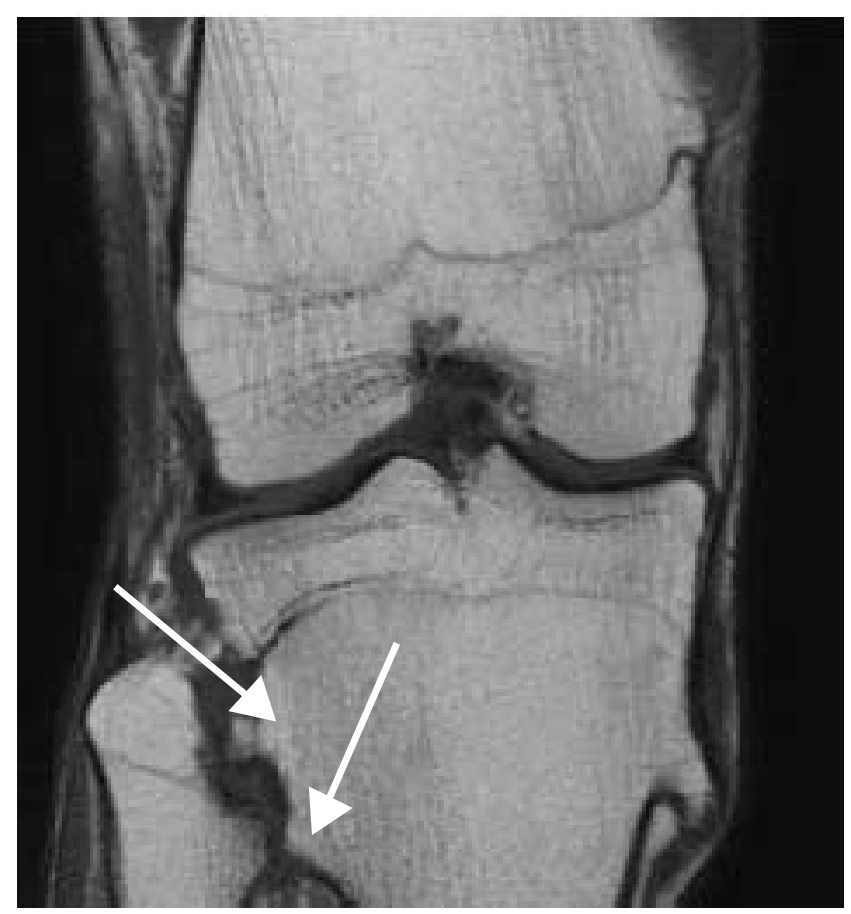

Fig-5: MRI of right knee demonstrates- exostosis in proximal part of both tibia and fibula.

The most striking feature is the presence of many exostoses, and disturbances in growth, such as abnormal tubulation of bones, producing broad and blunt metaphyses, and sometimes bowing of the radius and shortening of the ulna, producing ulnar deviation of the hand and valgus deformity of knee or ankle 9 . Most peroneal nerve trauma occurs at the fibular head, where the common peroneal nerve has not yet divided into its deep and superficial peroneal nerve and where most peroneal nerve lesions, therefore, involve both branches, although motor deficits are more frequently involved than sensory ones $^{4}$. Treatment for foot drop depends on the underlying cause $^{10}$. Specific treatment for foot drop may include physical therapy that strengthen leg muscles and help to maintain the range of motion in knee and ankle which may improve gait problems associated with foot drop. Stretching exercises are particularly important to prevent the development of stiffness of ankle. Ranges of motion exercises are for ankle and knee to maintain flexibility and mobility. Gait training is essential to walk with foot drop. Orthotics including Braces or splints that fits into the patients shoe can help holding foot in a normal position ${ }^{11}$. Electrical nerve stimulation (EST) stimulates dorsiflexor muscles to improve strength and reduce disuse atrophy. In cases where foot drop is relatively new, nerve surgery may be helpful.

\section{Conclusion}

The common peroneal nerve palsy is not very uncommon in case of hereditary multiple exostoses. Though surgery may be required to repair the damaged muscles, tendons and nerves to reduce the severity of the impairment the physiatric intervention plays an important role in the neurological recovery.

\section{References}

1. Beals RK, Horton W. Skeletal dysplasias: An approach to diagnosis. J Am Acad Orthop Surg 1995; 3:174-181

2. Porter et al., 2000. Porter DE, Emerton ME, Villanueva-Lopez F, et al: Clinical and radiographic analysis of osteochondromas and growth disturbance in hereditary exostoses.J Pediatr Orthop 2000; 20:246.

3. Siebenrock and Ganz, 2002. Siebenrock K-A, Ganz R: Osteochondroma of the femoral neck. Clin Orthop Relat Res 2002;394:211

4. Mnif H, Koubaa M, Zrig M, Zammel N, Abid A. Peroneal nerve palsy resulting from fibular head osteochondroma. Orthopedics. 2009;32(7):528

5. Carroll KL, Yandow SM, Ward K, Carey JC. Clinical correlation to genetic variations of hereditary multiple exostoses. J Pediatr Orthop 1999;19:785-791 6. Choi JH, Gu MJ, Kim MJ, et al. Fibrosarcoma in bizarre parosteal osteochondromatous proliferation. Skeletal Radiol 2001;30:44-47

7. Davids JR, Glancy GL, Eilert RE. Fracture through the stalk of pedunculated osteochondromas. A report of three cases. Clin Orthop Rel Res 1991;271:258-264.

8. El-Khoury GY, Bassett GS. Symptomatic bursa formation with osteochondromas. Am J Roentgenol 1979;133:895-898

9. Epstein DA, Levin EJ. Bone scintigraphy in hereditary multiple exostoses. Am J Roentgenol 1978;130:331-310

10. Mayo Clinic. Foot Drop. [Web site: www.mayoclinic.com/health/foot-drop/DS01031] 11. Stewart J. Eidelson and Susan Spinasanta. Spine Universe; Drop Foot

(Foot Drop) and Steppage Gait (Footdrop Gait); 2010 